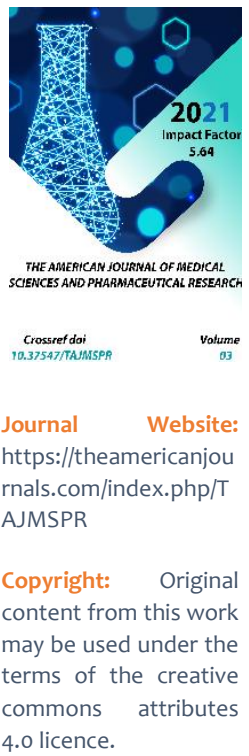

\title{
Characteristic Of The Morphometric Parameters Of The Liver In Polypragmasia
}

Usanov Sanat Sadinovich

Assistant Of The Department Of Anatomy And Clinical Anatomy, Samarkand State Medical Institute, Samarkand, Uzbekistan

\section{ABSTRACT}

We have studied the morphological, morphometric parameters of the liver of white rats. Against the background of polypharmacy, when using non-steroidal anti-inflammatory drugs, it was revealed in the structure of the liver, which led to various damage to the liver parenchyma. The simultaneous use of non-steroidal anti-inflammatory drugs leads to acute or chronic liver failure in rats. The longer the therapy for chronic diseases, the more pronounced morphological changes (necrosis, fibrosis, and cholestasis).

\section{KEYWORDS}

Liver, Polypharmacy, Non-Steroidal Therapy, Morphology, Morphomrytic Changes.

\section{INTRODUCTION}

In the modern world, there is a rapid growth in the creation and implementation of a huge number of medicines in reasonable health care, which, on the one hand, are capable of curing and improving the patient's condition, on the other, causing significant harm to health. The desire to improve the effectiveness of treatment, to help the patient recover from all the diseases that have developed in him, inevitably leads to the prescription of a large number of medicines (drugs) - polypharmacy (from the Greek. Poly - a lot, pragma - an object, thing). Polypharmacy is a serious health problem, as it clinically manifests itself in a decrease in the effectiveness of pharmacotherapy and the development of serious adverse reactions (ADRs), and a significant increase in health care costs. The term "polypharmacy" is often used in the medical literature, but there is no universally 
accepted definition. In domestic literary sources, polypharmacy is defined as the simultaneous prescription of many drugs, including their unjustified use. The term "polypharmacy" (polypharmacy, from the Greek poly-and pharmacy - medicine) is used in foreign literature. There is a qualitative definition of polypharmacy in other literary sources - the appointment of a patient with more drugs than the clinical situation requires and a quantitative definition - the appointment of 5 or more drugs to the patient $[1,2,5,7]$. In medical dictionaries, polypharmacy is defined as "mixing multiple drugs in one recipe," "using multiple drugs to treat one or more diseases; most often this phenomenon is observed in elderly patients," etc., and in scientific publications, there are more than 24 definitions $[14,15,16]$. The lack of consensus on the understanding of "polypharmacy" understandably leads to confusion among healthcare professionals. In recent years, there has been a clear trend towards an increase in the number of drug lesions due to the growing expansion of the pharmaceutical market. Thus, over 30 years in Japan, an 11-fold increase in drug hepatotoxicity was recorded $[6,7,8,9,9,10,11]$. More than 1200 drugs, 200 of which are potentially hepatotoxic, can cause the development of acute drug-induced liver damage (DIL). According to pharmacoepidemiological studies, DILI most often develops against the background of the use of non-steroidal anti-inflammatory drugs (NSAIDs), antimicrobial drugs, and drugs that affect the central nervous system (CNS), which is due not only to their potential hepatotoxicity but also to widespread use $[12,13,15.19] .40 \%$ of all cases of acute hepatitis in patients under the age of 40 and $13-25 \%$ of cases of development of fulminant hepatic failure (FPI) are due to drug hepatotoxicity $[2,4,8$,$] . Drug hepatitis$ complicates ongoing pharmacotherapy in 1$28 \%$ of cases, and $12-25 \%$ of cases contribute to the development of liver cirrhosis and liver failure. The most common hepatotoxic drugs include paracetamol with dose-dependent hepatotoxicity (in therapeutic doses, the drug is safe, if the daily dose of $4 \mathrm{~g}$ is exceeded, the likelihood of FPN development is high), NSAIDs, estrogens, anabolic steroids, antibiotics, anti-tuberculosis, antineoplastic drugs, cytostatics, phytopreparations. According to official US statistics, paracetamol causes $50 \%$ of FPN cases $[1,2,5,7]$.

\section{MATERIALS AND METHODS}

The studies were carried out on 50 Wistar rats weighing 180-200 g at 3-4 months. Pieces of the liver were taken for microscopic examination. Changes in the liver were studied after the use of NSAIDs at different periods of the experiment. The animals were slaughtered on days 3 and 30 of the experiment. To create experimental polypharmacy, the animals were injected intraperitoneally with NSAIDs (diclofenac, ibuprofen, dexamethasone) at a $0.4 \mathrm{mg} / \mathrm{kg}$ dose. The animals were slaughtered by decapitation in the morning. The material was fixed in a $10 \%$ neutral solution for $24 \mathrm{~h}$, dehydrated in a series of alcohols of increasing concentration, and embedded in paraffin. Paraffin blocks 5-7 $\mu \mathrm{m}$ thick were prepared using a sled micro ton. The sections were stained with hematoxylin-eosin.

\section{RESULTS}

We obtained the following microscopic changes: fatty degeneration of hepatocytes; foci of colliquation necrosis around the central veins with zonal necrosis. The third acini zone (centrilobular, perivenular) was also affected due to the high metabolic activity of this zone and the increased production of toxic 
metabolites when taking paracetamol. The inflammatory infiltrate consisted mainly of eosinophilic leukocytes (Figure 1).

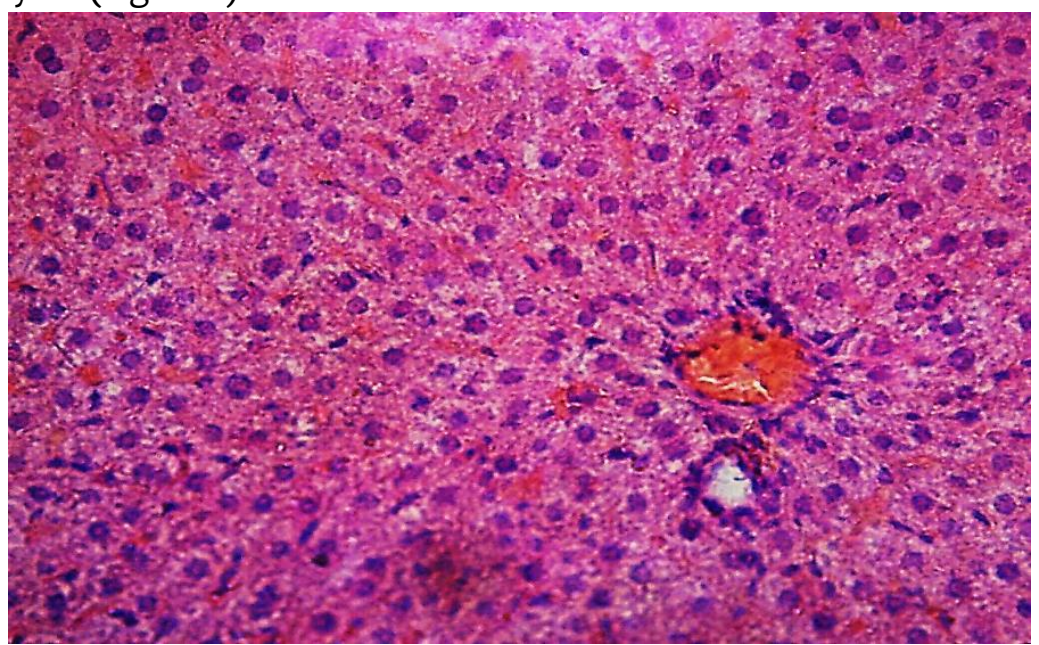

Fig. 1. In the liver stroma, pronounced edema, foci of colliquation necrosis around the central veins with zonal necrosis. Staining with hematoxylin and eosin. $10 \times 40$.



Fig. 2. When taking NSAIDs of the drug fatty degeneration of hepatocytes. Stained with hematoxylin and eosin, $10 \times 40$. 




Fig. 3. When taking NSAIDs, the drug destroys both the parenchyma and the stroma of the liverstained with hematoxylin and eosin, $10 \times 40$.

Revealed the defeat of the bile ducts with the development of dystrophic changes in their epithelium; cholestasis in the periportal lobules and vascular congestion, swollen hepatocytes (Fig. 3). Reconstruction of the liver structure with the formation of false lobules of a predominantly monolobular type, separated by fibrous septa.

\section{CONCLUSION}

Using NSAIDs simultaneously in a sufficient dosage (diclofenac and ibuprofen per day) in rats led to various damage to the liver parenchyma against the background of polypharmacy. Thus, the study results confirm that the simultaneous use of non-steroidal antiinflammatory drugs leads to acute or chronic liver failure in rats. The longer the therapy for chronic diseases, the more pronounced the morphological changes (necrosis, fibrosis, and cholestasis). Almost any drug of the NSAID group, including representatives of a new class, can cause liver damage and the development of NSAID hepatopathy against the background of polypharmacy.

\section{REFERENCES}

1. Baykova I.E., Nikitin I.G. (2009) Medicinal liver damage. RMJ (Russian Medical Journal), 1. pp. 4-10.

2. Bueverov A.O. (2009) Possibilities of treating drug-induced liver damage in conditions of the need to continue taking hepatotoxic drugs. Treat. physician, 2. Pp. 3-8.

3. Zvyagintseva T.D., Chernobay A.I. (2011) Medicinal hepatitis: from pathogenesis to treatment. Medicine and Pharmacy News, 375. pp. 23-28.

4. Zvyagintseva T.D., Chernobay A.I. (2011) Basic principles of treatment of chronic viral hepatitis. In the book: Chronic liver disease. Odessa, pp. 109153

5. Zvyagintseva T.D., Chernobay A.I. (2006) Alcohol-viral liver damage: the effectiveness of the new complex hepatoprotector "PM Sirin". Semeina medicine, 2. Pp. 44-48.

6. Klyuchareva A.A. (2007) Medicinal hepatitis. Medical News, 14. Pp. 19-24. 
7. Morozov Yu.A., Kucheryavyy Yu.A. (2011) Hepatoprotectors in clinical practice: rational aspects of use. A guide for doctors. 4TE Art., Moscow, p. 28.

8. Polunina T.E. (2013) Management of patients with drug-induced liver damage. Difficult Patient, 4. Pp. 8-13.

9. Kharchenko N.V., Anokhina G.A., Kharchenko V.V. (2013) Complex multifunctional hepatoprotectors in the treatment of patients with nonalcoholic liver disease in combination with hypertension. Practicing Likar, 1. Pp. 64-66.

10. Shirokova E.N. (2012) Medicinal lesions of the liver. Lecture at the All-Russian Internet Congress of Specialists in Internal Medicine. (internist.ruhttp: //www.internist.ru/articles/vnutrennie/ vnutrennie_671.html).

11. Shuba N.M., Voronova T.D. (2009) NSAID hepatotoxicity: is there a real threat? Ukr. rheumatol. zhurn., 1 (35). Pp. 33-39.

12. Aithal, G. P., Watkins, P. B., Andrade, R. J., Larrey, D., Molokhia, M., Takikawa, H., ... \& Daly, A. K. (2011). Case definition and phenotype standardization in drug-induced liver injury. Clinical Pharmacology \& Therapeutics, 89(6), 806-815.

13. Banks A., Zimmerman H., Isshak K., Harter J.G. (1995) Diclofenakassociated hepatotoxicity: an analysis of 180 cases report to the Food and Drug Administration as adverse reactions. Hepatology. 22 (3). Pp. 820870.

14. Bell, L. N., \& Chalasani, N. (2009, November). Epidemiology of idiosyncratic drug-induced liver injury.
In Seminars in liver disease (Vol. 29, No. 04, pp. 337-347). (c) Thieme Medical Publishers.

15. Bhogaraju, A., Nazeer, S., Al-Baghdadi, Y., Rahman, M., Wrestler, F., \& Patel, N. (1999). Diclofenac-associated hepatitis. Southern medical journal, 92(7), 711713.

16. Leitner, J. M., Graninger, W., \& Thalhammer, F. (2010). Hepatotoxicity of antibacterials: pathomechanisms and clinical data. Infection, 38(1), 3-11.

17. Galati, G., Tafazoli, S., Sabzevari, O., Chan, T. S., \& O'Brien, P. J. (2002). Idiosyncratic NSAID drug induced oxidative stress. Chemico-biological interactions, 142(1-2), 25-41.

18. Hughes, B. (2008). Industry concern over EU hepatotoxicity guidance. Nature Reviews Drug Discovery, 7(9), 719.

19. Leitner J. M., Graninger W., Thalhammer Infection., 38 (1). pp. 3-11.

20. Marschall H. U., Wagner M., Zollner G., Trauner M. (2007) Clinical hepatotoxicity. Regulation and treatment with inducers of transport and cofactors. Mol. pharm. 4 (6): 895910. 